\title{
Computation of antenna pattern correlation and MIMO performance by means of surface current distribution and spherical wave theory
}

\author{
O. Klemp, G. Armbrecht, and H. Eul \\ Universität Hannover, Institut für Hochfrequenztechnik und Funksysteme, Hannover, Germany
}

\begin{abstract}
In order to satisfy the stringent demand for an accurate prediction of MIMO channel capacity and diversity performance in wireless communications, more effective and suitable models that account for real antenna radiation behavior have to be taken into account. One of the main challenges is the accurate modeling of antenna correlation that is directly related to the amount of channel capacity or diversity gain which might be achieved in multi element antenna configurations. Therefore spherical wave theory in electromagnetics is a well known technique to express antenna far fields by means of a compact field expansion with a reduced number of unknowns that was recently applied to derive an analytical approach in the computation of antenna pattern correlation. In this paper we present a novel and efficient computational technique to determine antenna pattern correlation based on the evaluation of the surface current distribution by means of a spherical mode expansion.
\end{abstract}

\section{Introduction}

In this article the analysis of the multiple-input multipleoutput (MIMO) transmission behavior of antenna modules will be evaluated based upon an expansion of antenna surface current distribution represented by a series expansion involving spherical eigenmodes as in Stratton (1941) and Werner and Mittra (2000). The latter mathematical description yields a representation of antenna radiation patterns with a reduced order and therefore provides the advantage of an analytical approach in the description of multielement antenna transmission parameters. Therefore we present a modified technique of spherical mode expansion as in Chen et al. (1992) based upon a computation of vector expansion coefficients of the field approach that were directly derived by the surfacecurrent distribution on the antenna elements taken into con-

Correspondence to: O. Klemp (klemp@ hft.uni-hannover.de) sideration. Based upon a suitable statistical model to include for the spatial properties of a multipath propagation scenario as given in Jakes (1974), a closed form representation for antenna correlation may be derived as shown in Leifer (2002) and Klemp and Eul (2005). This representation of antenna correlation may be directly related to the computation of MIMO channel capacity as for the description of MIMO communication systems employing a certain number of antenna elements at the transmitter and at the receiver.

The computational models will be evaluated considering symmetrical planar, logarithmically-periodic four-arm antennas of different geometrical shapes that were designated for broadband operations in the frequency range between 1.0 GHz and 6.0 GHz as in Klemp et al. (2005). The antenna elements taken into consideration provide four symmetrical shaped and metalized antenna arms. Each adjacent pair of antenna arms represents one transmit- or receive branch of a MIMO-antenna system providing dual-linear polarization if fed with an opposite phase relation. Surface currents will be determined from appropriate computer models applying a conventional field simulator based on the finite-elementmethod (FEM). Applying simplified stochastic models of the radio channel that account for the spatio-temporal properties of multipath fading, we will extend our studies to the computation of MIMO channel capacities furthermore. The results of the applied current-based approach as well as the spherical mode expansion of the surface current distribution is accomplished according to Armbrecht et al. (2006) and compared to the standard methods given in Taga (1990) using a numerical integration in the far field domain of the considered antenna elements. Based on the results of convergence studies we will show the agreement between the different methods for the computation of antenna pattern correlation and MIMO channel capacity. The presented analysis establishes an explicit separation between antenna related properties and channel dependent quantities and could be therefore favorably applied to an optimization of antenna correlation

Published by Copernicus GmbH on behalf of the URSI Landesausschuss in der Bundesrepublik Deutschland e.V. 


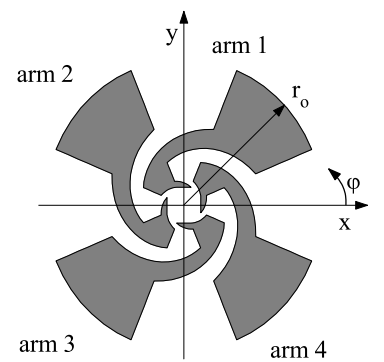

a) trapezoid antenna, $\mathrm{M}=1$

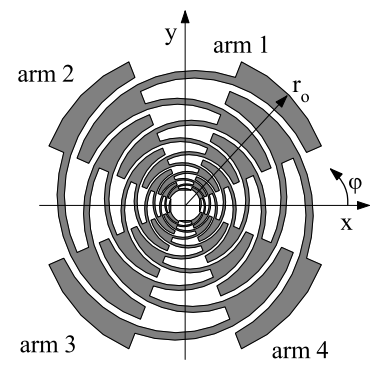

b) trapezoid antenna, $\mathrm{M}=5$
Fig. 1. Trapezoidal four-arm antenna elements with $M=1$ and $M=5$ periods of the trapezoidal unit cell.

or MIMO capacity. Based on the field expansion in terms of spherical eigenmodes, an investigation of the modal contributions to the overall antenna correlation can be performed additionally. In particular the analysis can be used to predict the MIMO performance of antenna elements in distinct channel scenarios by simply specifying their spectra of spherical eigenmodes.

The article is organized as follows, Sect. 2 summarizes the properties of self-complementary, four-arm, log.-per. planar antennas for applications with dual polarization. In Sect. 2.1 we review the fundamental properties of the spherical mode expansion technique with respect to the electric- and magnetic vector potential of a given elementary source distribution and apply this approach to the surface current distribution of the antenna elements taken into consideration. Section 3 is used to introduce the basic principles of MIMO transmission involving a channel structure based on the Kronecker model. Computing the modal representation of antenna radiation patterns as in Sect. 3.1 we derive an analytical approach for antenna power correlation based on the surface currents on the interactive antenna elements. The presented analysis is adopted to the computation of MIMO channel capacity in Sect. 4 for dual-polarized log.-per. planar four-arm antennas on each side of the communication link. Section 5 concludes this article.

\section{Antennas for broadband polarization diverse transmission}

Logarithmically-periodic antenna elements may theoretically provide frequency independent transmission characteristics (DuHamel and Isbell, 1957; Rumsey, 1957) if they adhere to the principle of self-complement. Designed as a planar logarithmically-periodic four-arm antenna element it offers dual linear polarization as shown in e.g. Klemp et al. (2005). Dual linear operation results from the odd-phase excitation of each of the two pairs of adjacent antenna arms. Antenna elements of that kind can be applied in a broadband antenna system for MIMO operations using dual orthogonal polarizations. In comparison to antenna configurations exploiting spatial diversity concepts, polarization diverse air interfaces provide independent branches for signal reception with a much smaller spatial extent.

In this paper, the planar, log.-per. four-arm trapezoid antenna as in Klemp et al. (2004) is used in order to demonstrate its capabilities as a compact polarization-diverse antenna element for MIMO communications based on an eigenmode characterization of its surface current distribution. Two different geometrical types of the trapezoid antenna will be investigated according to Fig. 1. Referring to Klemp et al. (2005) the trapezoid antennas taken into consideration are given by $M=1$ and $M=5$ periods of the trapezoidal unit cell that provides a trapezoidal slew rate of $50 \%$.

The outer radii of the antenna elements amount to $r_{\mathrm{O}}=50 \mathrm{~mm}$ and the antenna radiation behavior will be evaluated in free-space.

In order to derive a compact representation of antenna radiation behavior, the field quantities can be expanded by a compact set of spherical eigenmodes whose weights are computed from the appropriate current distribution on the antenna surface (e.g. Chen et al. (1992)). This field expansion yields a distinct set of vector expansion coefficients that is solely dependent on the excitation and the geometrical shape of the antennas taken into consideration. Therefore the spherical mode expansion may yield a significant order reduction in the description of antenna radiation behavior that speeds up computations of field-related quantities as e.g. the computation of antenna correlation and MIMO channel capacities. In Sect. 2.1 the results of the field expansion based on the surface current distribution are summarized.

\subsection{Spherical mode spectra of log.-per. antenna elements}

Following the procedure of spherical mode expansion for planar, log.-per. antenna elements based on its surface current distribution as given by Armbrecht et al. (2006), this section summarizes the spectra of spherical eigenmodes for the log.-per. trapezoid antennas with $M=1$ and $M=5$ periods of the trapezoidal unit cell as shown in Fig. 1.

As an example, Fig. 2 shows the normalized SME coefficients at a simulation frequency of $3 \mathrm{GHz}$. The normalization is performed with respect to the absolute value of the expansion coefficient of degree $m=0$ and order $n=0, \underline{\boldsymbol{a}}_{00}$, which yields normalized weights $\left\|\underline{\boldsymbol{a}}_{n m}\right\| /\left\|\underline{\boldsymbol{a}}_{00}\right\|$ in terms of expansion coefficients related to even spherical harmonics $Y_{n m}^{e}(\vartheta, \varphi)$ and $\left\|\underline{\boldsymbol{b}}_{n m}\right\| /\left\|\underline{\boldsymbol{a}}_{00}\right\|$ for expansion coefficients of odd spherical harmonics $Y_{n m}^{o}(\vartheta, \varphi)$ (see Stratton, 1941).

As can be seen from the decaying behavior of modal contributions with increasing degree and order of the harmonics, it becomes feasible to limit the number of modes in the field expansion with respect to a finite value $N$. Therefore it is advantageous to conduct a radiation pattern expansion by spherical eigenmodes because the derived set of expansion coefficients yields an approximative low-order characterization of the radiation profile of the related antenna structure. 


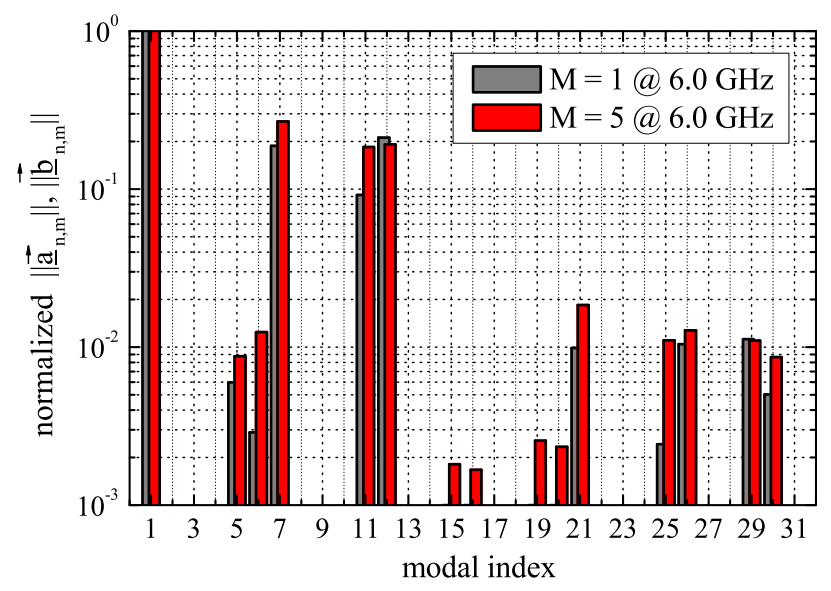

Fig. 2. Normalized coefficients of the spherical mode expansion for trapezoid antennas $M=1$ and $M=5$ at $3 \mathrm{GHz}$. See Armbrecht et al. (2006) for the appropriate enumeration of the abscissa.

\section{Multiple-input multiple-output antenna systems}

Multiple-input multiple-output antenna systems may efficiently exploit the spatial domain by parallel subchanneling employing decoupled transmit- and receive paths as shown in Foschini (1996), Yu and Ottersten (2002). Due to the ability of transmitting simultaneous data streams incorporating antenna arrays at both sides of the radio link, a great increase of channel capacities in wireless communications may be achieved. The establishment of parallel subchannels is a result of uncorrelated fading processes between individual transmission paths of the communication link and can be a result of spatial-, pattern- or polarization diverse properties of the applied antenna elements. Depending on the properties of the regarded multipath channel, the application of dual-polarized antenna modules at the receiver and at the transmitter is possible. Compared to MIMO techniques exploiting spatial diversity concepts, using polarization-diverse air interfaces leads to the design of compact transmission terminals with enhanced transmission capacity.

Figure 3 shows the model of the considered wireless MIMO system incorporating log.-per. antenna elements on both sides of the radio link. The four-arm antenna structures provide orthogonal-polarized operational modes by feeding one of the two adjacent antenna arm pairs with an odd phase relation. Using one antenna element with dual linear polarization at the transmit (TX) side and one antenna element with dual linear polarization at the receive (RX) side yields a $(2 \times 2)$-MIMO communication system.

Given frequency flat signal vectors of the TX- and the RX antenna array, $\mathbf{x}$ and $\mathbf{y}$, the channel matrix equation of the corresponding frequency flat channel following Yu and Ottersten (2002) is given as follows:

$\mathbf{y}=\mathbf{H} \mathbf{x}+\mathbf{n}$.

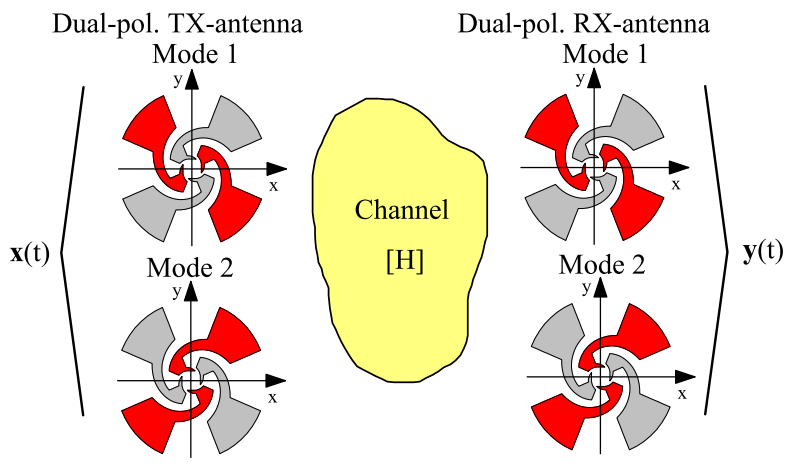

Fig. 3. Model of the MIMO radio channel incorporating $n^{\mathrm{TX}}$ antennas at the TX-side and $n^{\mathrm{RX}}$ antennas at the $\mathrm{RX}$-side.

$\mathbf{H}$ is the narrowband transmission matrix of the MIMO channel and $\mathbf{n}$ describes zero-mean additive white Gaussian noise with a variance of $\sigma_{n}^{2}$. The complex correlation coefficient between independent operational modes $i$ and $j$ at the transmitter- or at the receiver side is given in accordance with Taga (1990) for incident phase centers as:

$\rho_{\mathrm{i}, \mathrm{j}}^{\mathrm{TX}, \mathrm{RX}}=\frac{R_{\mathrm{i}, \mathrm{j}}^{\mathrm{TX}, \mathrm{RX}}}{\sqrt{\sigma_{\mathrm{i}}^{\mathrm{TX}, \mathrm{RX}^{2}} \sigma_{\mathrm{j}}^{\mathrm{TX}, \mathrm{RX}^{2}}}}$.

From Eq. (2) antenna correlation $\rho_{\mathrm{i}, \mathrm{j}}^{\mathrm{TX}, \mathrm{RX}}$ depends on antenna covariance $R_{\mathrm{i}, \mathrm{j}}^{\mathrm{TX}, \mathrm{RX}}$ and the variances $\sigma_{\mathrm{i}}^{\mathrm{TX}, \mathrm{RX}^{2}}$ and $\sigma_{\mathrm{j}}^{\mathrm{TX}, \mathrm{RX}{ }^{2}}$. $R_{\mathrm{i}, \mathrm{j}}$ can be computed from the radiation patterns $\boldsymbol{C}(\vartheta, \varphi)$ of operational mode $i$ and operational mode $j$, as follows:

$$
\begin{aligned}
& R_{i, j}=\mathrm{K} \int_{0}^{2 \pi} \int_{0}^{\pi}\left[C_{\vartheta, \mathrm{i}}(\vartheta, \varphi) C_{\vartheta, \mathrm{j}}^{*}(\vartheta, \varphi)\right. \\
& \left.+\operatorname{XPD} C_{\varphi, \mathrm{i}}(\vartheta, \varphi) C_{\varphi, \mathrm{j}}^{*}(\vartheta, \varphi)\right] p_{\vartheta, \varphi}(\vartheta, \varphi) \sin \vartheta d \vartheta d \varphi .
\end{aligned}
$$

In Eq. (3), $p_{\vartheta, \varphi}(\vartheta, \varphi)$ denotes a two-dimensional probability density function that accounts for the spatial property of outgoing waves at the transmitter and impinging waves at the receiver. The ratio of the mean incident power in vertical direction to the mean incident power in horizontal direction at the locations of receiver and transmitter is given by the crosspolarization discrimination XPD. ${ }^{*}$, means conjugate operation and $K$ is a proportionality constant. Replacing $i=j$ in Eq. (3) yields the variances of the operational modes $i$ and $j$, $\sigma_{\mathrm{i}}^{\mathrm{TX}, \mathrm{RX}^{2}}, \sigma_{\mathrm{j}}^{\mathrm{TX}, \mathrm{RX}^{2}}$, respectively.

The correlation values between any possible combination of adjacent port-related antenna patterns at the TX- and at the RX-side will be combined to correlation matrices $\mathbf{R}^{\mathrm{TX}}$ and $\mathbf{R}^{\mathrm{RX}}$ (Kermoal et al., 2002). Performing the Kroneckerproduct between the correlation matrices, $\mathbf{R}^{\mathrm{TX}} \otimes \mathbf{R}^{\mathrm{RX}}$ yields the correlation properties of the channel matrix $\mathbf{H}$ of the communication link. In order to determine the theoretical channel capacity of the proposed MIMO radio link, Monte-Carlo simulations are performed, involving $N_{\mathbf{H}}=5000$ realizations 
of $\mathbf{H}$. Initially, the elements of $\mathbf{H}$ are assumed to be uncorrelated zero-mean complex Gaussian variables with a unit variance. The appropriate correlation values between the channel coefficients are computed from matrix multiplication with the correlation matrix $\mathbf{R}^{\mathrm{TX}} \otimes \mathbf{R}^{\mathrm{RX}}$ as shown in Kermoal et al. (2002).

Provided a uniform power allocation between the independent operational modes at the transmit antenna, the ergodic MIMO channel capacity $\mathrm{s}$ given as a sum over $K$ subchannels:

$C=\sum_{k=1}^{K} \log _{2}\left(1+\lambda_{\mathrm{k}} \frac{P_{\mathrm{k}}}{\sigma_{n}^{2}}\right)$.

In Eq. (4), $P_{\mathrm{k}}$ denotes the amount of power assigned to the $k$ th subchannel and $\sigma_{n}^{2}$ is the total noise power at the receiver. $\lambda_{\mathrm{k}}$ gives the eigenvalue of the $k$-th subchannel.

\subsection{SME-based computation of antenna pattern correlation}

In order to simplify the computation of antenna pattern correlation and MIMO channel capacity which are directly related to the radiation patterns of the involved antenna elements as shown in Sect. 3, a spherical mode expansion of the surface current distribution will be accomplished. Due to the fact, that the radiation patterns of each antenna element may be described by a distinct set of modal expansion coefficients this analysis is predestined to provide a deeper insight into the radiation mechanisms that cause high values of pattern correlation and low channel capacities. Furthermore it will become possible to optimize delimiting antenna geometries with respect to certain cost functions of related transmission parameters that take into account the real antenna radiation behavior.

As shown in Armbrecht et al. (2006), antenna radiation patterns $\boldsymbol{C}(\vartheta, \varphi)$ of an arbitrary antenna geometry can be expressed by an expansion of the current distribution on the respective antenna geometry by means of an orthogonal set of spherical basis functions as shown in Eq. (5). The subsequent analysis is based on planar antenna geometries, whose metalization surfaces are located in parallel to the $x y$-plane of a cartesian coordinate system. Surface current distributions $\boldsymbol{J}\left(x^{\prime}, y^{\prime}\right)=\left[J_{x}\left(x^{\prime}, y^{\prime}\right), J_{y}\left(x^{\prime}, y^{\prime}\right)\right]$ (where $x^{\prime}$ and $y^{\prime}$ indicate the cartesian coordinates of the source region) are derived using a conventional full-wave simulator based on the finite-element-method (FEM). Assuming far-field conditions of the electromagnetic field, the spherical mode expansion of the surface current distribution yields:

$$
\begin{aligned}
{\left[\begin{array}{l}
C_{\vartheta, i} \\
C_{\varphi, i}
\end{array}\right]=} & -j k \eta \sum_{n=0}^{\infty} \sum_{m=0}^{n} j^{n}\left[\begin{array}{cc}
\cos \vartheta \cos \varphi \cos \vartheta \sin \varphi \\
-\sin \varphi & \cos \varphi
\end{array}\right] \\
& {\left[\begin{array}{cc}
\underline{a}_{n_{i} m_{i}}^{x, i} & \underline{b}_{n_{i} m_{i}}^{x, i} \\
\underline{a}_{n_{i} m_{i}}^{y, b} & \underline{b}_{n_{i} m_{i}}^{y, i}
\end{array}\right]\left[\begin{array}{c}
Y_{n_{i} m_{i}}^{e}(\vartheta, \varphi) \\
Y_{n_{i} m_{i}}^{o}(\vartheta, \varphi)
\end{array}\right] . }
\end{aligned}
$$

In Eq. (5), spherical harmonics $Y_{n m}^{e}(\vartheta, \varphi)$ and $Y_{n m}^{e}(\vartheta, \varphi)$ are mapped to the spherical components $C_{\vartheta, i}, C_{\varphi, i}$ of an- tenna radiation pattern $i$ incorporating a field expansion matrix including vector expansion coefficients $\underline{\boldsymbol{a}}_{n_{i} m_{i}}^{i}, \underline{\boldsymbol{b}}_{n_{i} m_{i}}^{i}$ as derived from the surface current distribution of operational mode $i$ and an additional matrix that accounts for coordinate transformation. The coordinate transform of the cartesian components of the surface currents $J_{x}\left(x^{\prime}, y^{\prime}\right)$ and $J_{y}\left(x^{\prime}, y^{\prime}\right)$ into spherical components is mandatory in order to apply the Fraunhofer approximation of the electromagnetic field (Lo and Lee, 1988). For convenience, in Eq. (5), the upper infinite summation index of the spherical eigenfield decomposition has to be replaced by a finite value $N$ leading to impairments in the synthesized far-field representation. Those impairments can be reduced by adhering to the convergence criteria for spherical wave expansions as in Narasimhan et al. (1985). Therefore a sufficient degree of field approximation is derived for $N \geq k a, a$ denoting the radius of a sphere that is completely enclosing the considered antenna and $k$ is the scalar wave number.

Splitting the integral for covariance computation $R_{i, j}$ as given in Eq. (3) into two parts, $R_{i, j}^{\vartheta}$ and $R_{i, j}^{\varphi}$ that account for the contributions of antenna covariance in the directions of $\vartheta$ and $\varphi$ and replacing $\boldsymbol{C}(\vartheta, \varphi)$ by the eigenfield decomposition as given in Eq. (5), it follows:

$$
\begin{aligned}
& R_{i, j}^{\vartheta, \varphi}=\mathrm{K} \sum_{n_{i}=0}^{\infty} \sum_{m_{i}=0}^{n_{i}} \sum_{n_{j}=0}^{\infty} \sum_{m_{j}=0}^{n_{j}} j^{n_{i}} j^{n_{j}^{*}} \\
& \left\{\mathbf{T}^{e e}\left[\begin{array}{c}
I_{n_{i} m_{i}, n_{j} m_{j}}^{e e, M^{\vartheta, \varphi}} \\
I_{n_{i} m_{i}, n_{j} m_{j}}^{e e, M_{j}^{\vartheta, \varphi}} \\
I_{n_{i} m_{i}, n_{j} m_{j}}^{e e, M_{3, \varphi}^{\vartheta, \varphi}}
\end{array}\right]+\mathbf{T}^{e o}\left[\begin{array}{c}
I_{n_{i} m_{i}, n_{j} m_{j}}^{e o, M_{j}^{\vartheta, \varphi}} \\
I_{n_{i} m_{i}, n_{j} m_{j}}^{e o, M_{j}^{\vartheta, \varphi}} \\
I_{n_{i} m_{i}, n_{j} m_{j}}^{e o, M_{j}^{\vartheta, \varphi}}
\end{array}\right]\right. \\
& \left.+\mathbf{T}^{o e}\left[\begin{array}{c}
o e, M_{1}^{\vartheta, \varphi} \\
I_{n_{i} m_{i}, n_{j} m_{j}} \\
I_{n_{i} m_{i}, n_{j} m_{j}}^{o e, m^{\vartheta}, \varphi} \\
I_{n_{i} m_{i}, n_{j} m_{j}}^{o e, M^{\vartheta, \varphi}}
\end{array}\right]+\mathbf{T}^{o o}\left[\begin{array}{c}
o o, M_{1}^{\vartheta, \varphi} \\
I_{n_{i} m_{i}, n_{j} m_{j}} \\
I_{n_{i} m_{i}, n_{j} m_{j}}^{o o, m_{j}} \\
I_{n_{i} m_{i}, n_{j} m_{j}}^{o o, M^{\vartheta, \varphi}}
\end{array}\right]\right\} \text {. }
\end{aligned}
$$

In Eq. (6), $\quad I_{n_{v} m_{v}, n_{\mu} m_{\mu}}^{e, o e, o M_{\xi}^{\vartheta}}$ (with $v=i, j, \quad \mu=i, j$ and $\xi=1, \ldots, 3)$ denote coupling integrals between two spherical harmonics $Y_{n_{j} m_{j}}^{e, o}(\vartheta, \varphi)$ in a distinct channel scenario $p_{\vartheta, \varphi}(\vartheta, \varphi)$, weighed by a field-point dependent factor $M_{\xi}^{\vartheta}(\vartheta, \varphi)$ as follows:

$$
\begin{aligned}
& \left\{\begin{array}{c}
I_{n_{v} m_{v}, n_{\mu} m_{\mu}}^{e, o e, o, M_{\xi}^{\vartheta}} \\
I_{n_{v} m_{v}, n_{\mu} m_{\mu}}^{e, o e, M_{\xi}^{\varphi}}
\end{array}\right\}=\int_{\varphi=0}^{2 \pi} \int_{\vartheta=0}^{\pi}\left\{\begin{array}{l}
M_{\xi}^{\vartheta}(\vartheta, \varphi) \\
M_{\xi}^{\varphi}(\vartheta, \varphi)
\end{array}\right\} \\
& Y_{n_{i} m_{i}}^{e, o}(\vartheta, \varphi) Y_{n_{j} m_{j}}^{e, o}(\vartheta, \varphi) p_{\vartheta, \varphi}(\vartheta, \varphi) \sin \vartheta d \vartheta d \varphi .
\end{aligned}
$$

The antenna-dependent scaling factors $\mathbf{T}^{e e}, \mathbf{T}^{e o}, \mathbf{T}^{o e}$ and $\mathbf{T}^{o o}$ in Eq. (6) are given entirely with respect to the field expansion coefficients:

$$
\mathbf{T}^{e e}=\left[\begin{array}{c}
\underline{a}_{n_{i} m_{i}}^{x, i} \underline{a}_{n_{j} m_{j}}^{x, j^{*}} \\
\underline{a}_{n_{i} m_{i}}^{x} \underline{a}_{n_{j} m_{j}}^{y^{*}}+\underline{a}_{n_{i} m_{i}}^{y, i} \underline{a}_{n_{j} m_{j}}^{x, j^{*}} \\
\underline{a}_{n_{i} m_{i}}^{y, i} \underline{a}_{n_{j} m_{j}}^{y, j^{*}}
\end{array}\right],
$$




$$
\begin{aligned}
& \mathbf{T}^{e o}=\left[\begin{array}{c}
\underline{a}_{n_{i} m_{i}}^{x, i} \underline{b}_{n_{j} m_{j}}^{x, j^{*}} \\
\underline{a}_{n_{i} m_{i}}^{x} \underline{b}_{n_{j} m_{j}}^{y^{*}}+\underline{b}_{n_{n^{\prime} m_{i}}}^{y, a_{n_{j} m_{j}}^{x, j^{*}}} \\
\underline{a}_{n_{i} m_{i}}^{y, i} \underline{a}_{n_{j} m_{j}}^{y, m^{*}}
\end{array}\right], \\
& \mathbf{T}^{o e}=\left[\begin{array}{c}
\underline{b}_{n_{i} m_{i}}^{x, i} a_{n_{j} m_{j}}^{x, j^{*}} \\
\underline{b}_{n_{i} m_{i}}^{x} \underline{a}_{n_{j} m_{j}}^{y^{*}}+\underline{b}_{n_{i}}^{y, i} m_{i} \underline{a}_{n_{j} m_{j}}^{x, j^{*}} \\
\underline{b}_{n_{i} m_{i}}^{y, i} \underline{a}_{n_{j} m_{j}}^{y, m^{*}}
\end{array}\right]
\end{aligned}
$$

and

$$
\mathbf{T}^{o o}=\left[\begin{array}{c}
\underline{b}_{n_{i}}^{x, i} \underline{b}_{n_{j}}^{x, j^{*}} \\
\underline{b}_{n_{i} m_{i}}^{x} \underline{b}_{n_{j} m_{j}}^{y^{*} m_{j}}+\underline{b}_{n_{j} m_{i}}^{y, j} \underline{b}_{n_{j} m_{j}}^{x, j^{*}} \\
\underline{b}_{n_{i} m_{i}}^{y, i} \underline{b}_{n_{j} m_{j}}^{y, m^{*}}
\end{array}\right] .
$$

The field-point dependent factors $M_{\xi}^{\vartheta}(\vartheta, \varphi)$ related to the $\vartheta$ component of the antenna radiation pattern can be computed from:

$$
\left\{\begin{array}{l}
M_{1}^{\vartheta}(\vartheta, \varphi) \\
M_{2}^{\vartheta}(\vartheta, \varphi) \\
M_{3}^{\vartheta}(\vartheta, \varphi)
\end{array}\right\}=\left\{\begin{array}{c}
\left.\cos ^{2} \vartheta \cos ^{2} \varphi\right) \\
\cos ^{2} \vartheta \cos \varphi \sin \varphi \\
\cos ^{2} \vartheta \sin ^{2} \varphi
\end{array}\right\} .
$$

The scaling factors $M_{\xi}^{\varphi}(\vartheta, \varphi)$ that are related to the $\varphi$ component of $\boldsymbol{C}(\vartheta, \varphi)$ yield:

$$
\left\{\begin{array}{l}
M_{1}^{\varphi}(\vartheta, \varphi) \\
M_{2}^{\varphi}(\vartheta, \varphi) \\
M_{3}^{\varphi}(\vartheta, \varphi)
\end{array}\right\}=\left\{\begin{array}{c}
\sin ^{2} \varphi \\
-\cos \varphi \sin \varphi \\
\cos ^{2} \varphi
\end{array}\right\} .
$$

As can be seen from Eq. (6), a complete separation between antenna- and channel dependent quantities is achieved. It is also an important advantage that the values of the coupling integrals in Eq. (7) remain fixed within a distinct channel scenario $p_{\vartheta, \varphi}(\vartheta, \varphi)$. Due to the fact that antenna radiation behavior is characterized in terms of an SME by its vector field expansion coefficients $\underline{\boldsymbol{a}}_{n_{i} m_{i}}^{i}$ and $\underline{\boldsymbol{b}}_{n_{i} m_{i}}^{i}$, the effect of changing the antenna topology or geometry of single antenna elements within the considered channel scenario can be easily obtained from Eq. (6).

\subsection{Correlation properties of log.-per. four-arm antennas}

In this section the correlation properties of log.-per. antenna elements at the transmitter or at the receiver are investigated. The analysis is accomplished using the novel approach for the computation of power correlation as derived in Sect. 3.1 based on the SME expansion of the surface current distributions of the related antenna elements. Results were computed in terms of a two-dimensional probability density $p_{\vartheta, \varphi}(\vartheta, \varphi)$ applying a Laplacian dependence in azimuth $\varphi$ and a Gaussian dependence in elevation $\vartheta$ in accordance to Taga (1990) featuring mean values $m_{\varphi}=90^{\circ}, m_{\vartheta}=90^{\circ}$ and angular spreads $\sigma_{\varphi}=10^{\circ}, \sigma_{\vartheta}=10^{\circ}$.

Antenna power correlation is computed between the vector antenna radiation patterns $\boldsymbol{C}_{i, j}(\vartheta, \varphi)$ related to the operational modes $i$ and $j$ of the antenna element with dual linear

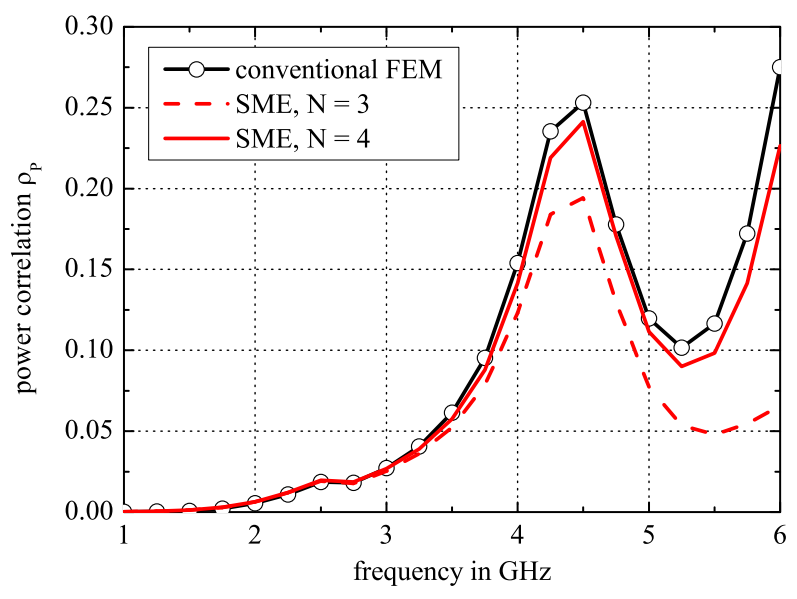

Fig. 4. Power correlation between orthogonal operational modes for trapezoid antenna $M=1$.

polarization. Figure 4 shows the results for antenna power correlation $\rho_{P}$ evaluated by the standard approach involving electric far-fields derived by methods of FEM (see Eq. (3)) and a spherical mode expansion of the surface-current distribution. Results are shown in the predestined frequency range of the antenna elements between $1 \mathrm{GHz}$ and $6 \mathrm{GHz}$. Increasing the order of the field approach ameliorates the fit between the two computational approaches (FEM and SME) as can be seen from Fig. 4. Therefore including a maximum order $N=4$ seems adequate for the trapezoid antenna $M=1$ that is depicted in Fig. 1a. Due to the imperfect polarization decoupling between the two operational modes of this antenna element, $\rho_{P}$ yields a peak at the frequency of $4.3 \mathrm{GHz}$ and a local maximum at the upper frequency of $6 \mathrm{GHz}$ of the considered frequency range. According to the trapezoid antenna including $M=5$ periods of the trapezoidal unit cell as depicted in Fig. 1b, the results of power correlation $\rho_{P}$ are shown in Fig. 5. Due to excellent polarization decoupling between the two pairs of adjacent antenna arms for this antenna element, power correlation remains below 0.015 in the entire frequency range. Despite those low numerical values for $\rho_{P}$ the results derived by spherical eigenfield decomposition of the surface current distribution including a maximum expansion degree $N=3$ are in an excellent agreement.

\section{Analysis of MIMO antenna performance by means of SME}

Following the stochastic model given in Sect. 3 for a $(2 \times 2)$ MIMO antenna configuration using identical trapezoid antenna elements with dual-linear polarization at the transmitter and at the receiver, the ergodic channel capacities were computed from Eq. (4). In order to evaluate the applicability of the spherical wave approach as given in Sect. 3.1 in terms of MIMO transmission performance, the results for transmission capacity were plotted for the spherical wave approach 


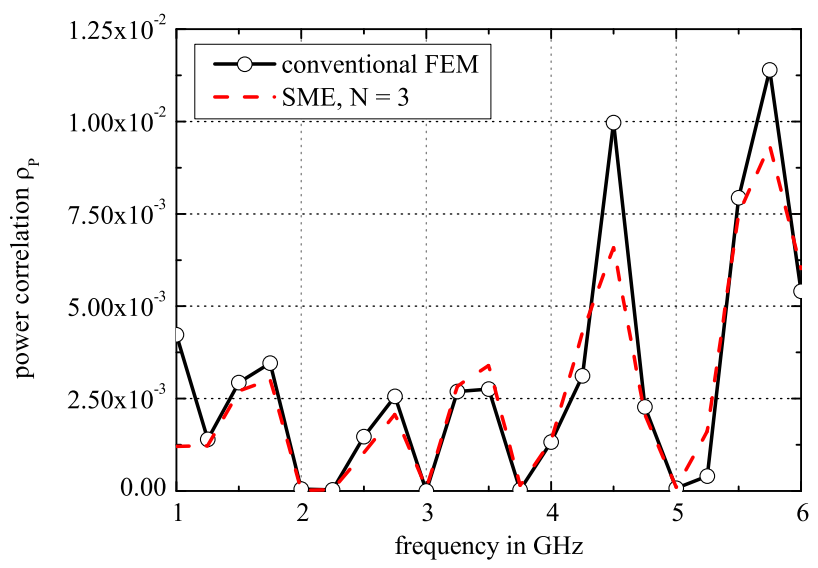

Fig. 5. Power correlation between orthogonal operational modes for trapezoid antenna $M=5$.

in comparison to the conventional, FEM-based results. The results in this section are limited to a maximum degree of $N=3$ for the spherical wave expansion.

Figures 6 and 7 show the results for MIMO channel capacity in terms of cumulative distribution functions (CDFs) of the outage capacity in bit $/ \mathrm{s} / \mathrm{Hz}$ for the trapezoid antenna $M=1$. At $3 \mathrm{GHz}$, the results of conventional FEMbased computation and spherical mode expansion are in a very good agreement, as can be seen from Fig. 6. Regarding the sum capacity for the $(2 \times 2)$-MIMO transmission link, both computational techniques consistently yield a $10 \%$-outage capacity of $10.45 \mathrm{bit} / \mathrm{s} / \mathrm{Hz}$. At a simulation frequency of $6 \mathrm{GHz}$, where slight deviations between the two computational techniques were reported in terms of antenna power correlation $\rho_{P}$ (see Fig. 4), the results for conventional computed channel capacity and channel capacity as derived by means of SME $(N=3)$ are not in an excellent agreement. Following the values for the 10\%-outages of the channel capacity, spherical mode expansion exhibits a slight overestimation of the channel capacity resulting in $C_{10 \%, 6 \mathrm{GHz}}=10.4 \mathrm{bit} / \mathrm{s} / \mathrm{Hz}$. Conventional, FEM-based computations yield a value of $10.2 \mathrm{bit} / \mathrm{s} / \mathrm{Hz}$. Especially the CDF for the weaker channel of the transmission link can not be approximated very well by means of SME. This result is due to the small contributions of this spatial subchannel to the overall capacity of the MIMO link, where impairments in the field-representations due to an inadequate degree of modal field expansion become visible first.

This result is in a close agreement to the values for antenna power correlation at $6 \mathrm{GHz}$ as shown in Fig. 4. In this case, SME yields a power correlation of 0.07 whereas conventional, FEM-based computations lead to $\rho_{P}=0.28$. As can be seen from these results, frequency variation of the ergodic channel capacity is extremely low.

The results for using trapezoid antennas with $M=5$ periods of the trapezoidal unit cell (see Fig. 1b) in a $(2 \times 2)$ MIMO communication link are depicted in Fig. 8 at $3 \mathrm{GHz}$

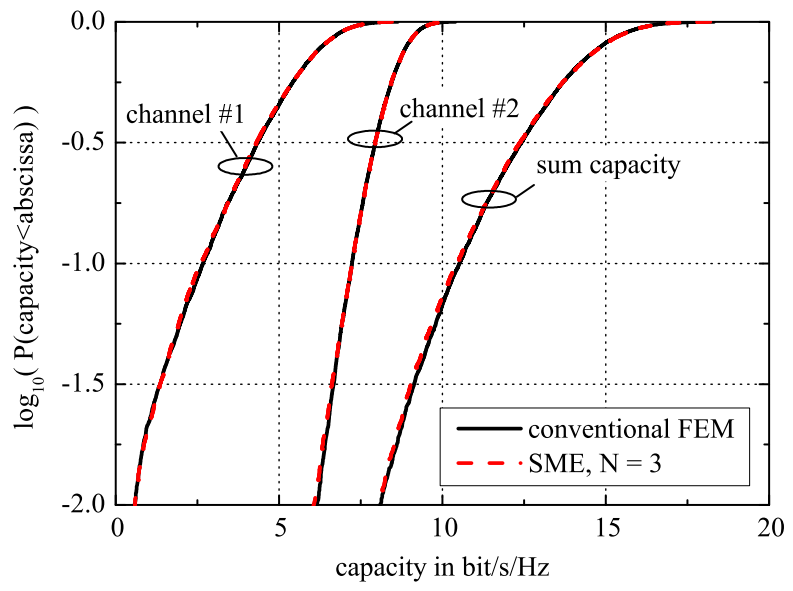

Fig. 6. CDF of MIMO channel capacity for $M=1$ antenna at $3 \mathrm{GHz}$ derived from FEM computations and SME, $N=3$.

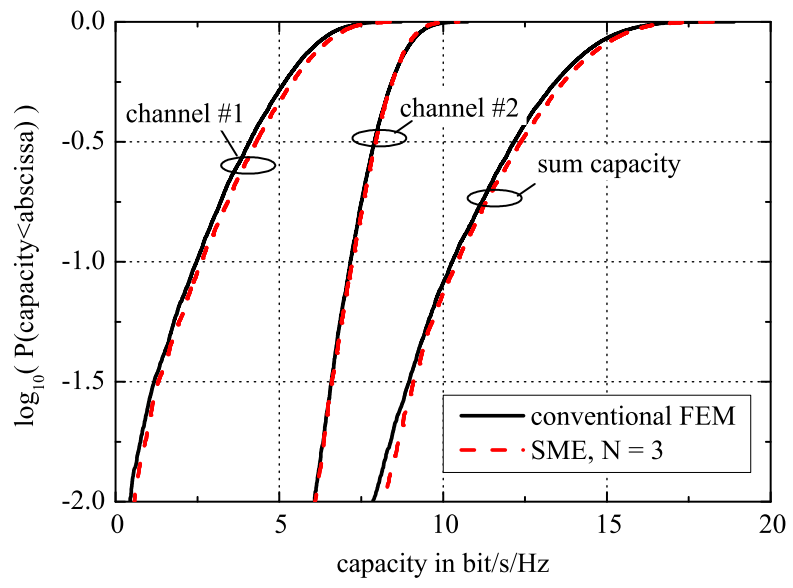

Fig. 7. $\mathrm{CDF}$ of MIMO channel capacity for $M=1$ antenna at $6 \mathrm{GHz}$ derived from FEM computations and SME, $N=3$.

and in Fig. 9 for a simulation frequency of $6 \mathrm{GHz}$, respectively. As already shown for the trapezoid antenna $M=1$, frequency variation of the ergodic channel capacity is found to be very low. The approximations of the CDFs for the channel capacities based on the SME of the surface current distributions are in a very close agreement to the results as derived from the conventional FEM-based computations. As shown in Fig. 8 , at $3 \mathrm{GHz}$, the $10 \%$-outage capacity based on FEMresults indicates a value of $10.5 \mathrm{bit} / \mathrm{s} / \mathrm{Hz}$ that is in exact agreement with the results based on the spherical mode expansion $(N=3)$. At a simulation frequency of $6 \mathrm{GHz}$ FEM-based computations yield a value of $10.5 \mathrm{bit} / \mathrm{s} / \mathrm{Hz}$ for the $10 \%$ outage capacity that is in a good agreement to the result for SME-based computations that yields a value of $10.4 \mathrm{bit} / \mathrm{s} / \mathrm{Hz}$. Due to the approximation of Rayleigh distributed signal fading by a finite occurrence of 5000 channel realizations as shown in Sect. 3 this result remains within computational exactness of the applied model of the radio channel. 


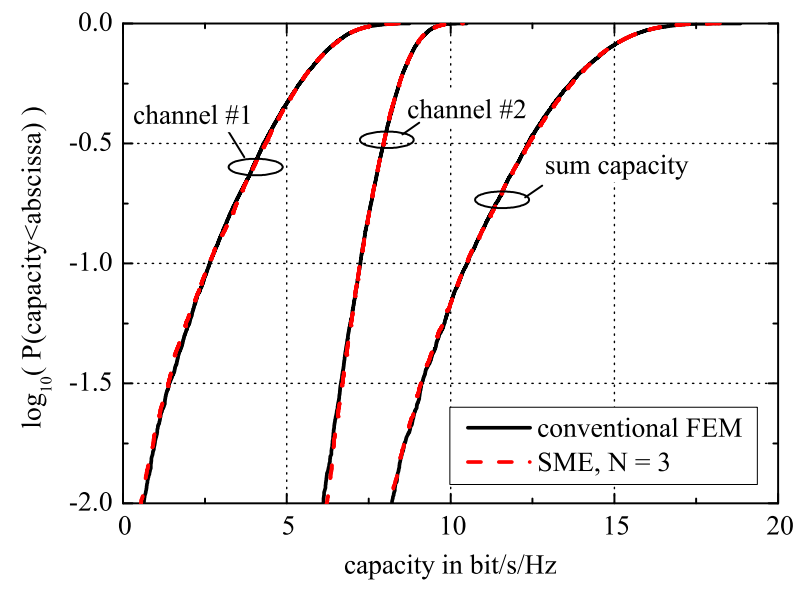

Fig. 8. CDF of MIMO channel capacity for $M=5$ antenna at $3 \mathrm{GHz}$ derived from FEM computations and SME, $N=3$.

\section{Conclusions}

This paper presented a generalized approach for the computation of antenna power correlation and MIMO channel capacity in multielement antenna arrangements based on a spherical mode expansion of the respective surface current distributions. The analysis was applied in order to characterize the power correlation and channel capacity for planar, dual-polarized log.-per. four-arm antenna elements. The characterization of the MIMO radio channel was based on a simple stochastic model that accounted for the spatiotemporal behavior of signal fading at the transmitter and at the receiver. Results based on the introduced spherical mode expansion were found to be in a very close agreement to the results as derived by conventional FEM-based computations. The major advantages of this work is the complete analytical description of the link-related transmission parameters like antenna power correlation and channel capacity by means of spherical harmonics. Due to the fact that the coefficients of field expansion that are directly related to the surface current distribution on the related antenna elements are separated mathematically from the quantities that characterize the channel model, this approach allows a clearer insight into the field-related properties of MIMO communications systems. It may be additionally used in order to optimize or fit certain antenna geometries to distinct channel scenarios.

\section{References}

Armbrecht, G., Klemp, O., and Eul, H.: Spherical Mode Analysis of Planar Frequency-Independent Multi-Arm Antennas Based on Its Surface Current Distribution, Accepepted for publ. in Adv. Radio Sci., 2006.

Chen, Y., Simpson, T. L., and Ho, T. Q.: Highly efficient technique for solving radiation and scattering problems, IEE Proc.-H, 139, 7-10, 1992.

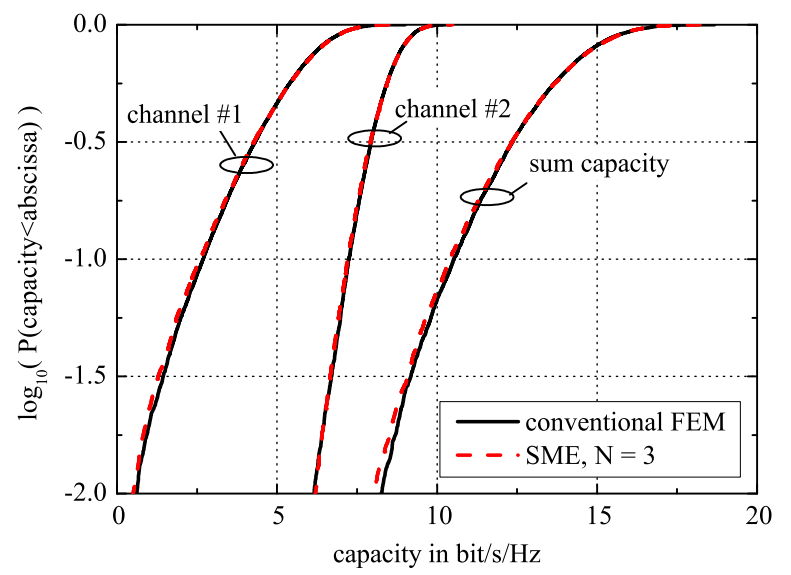

Fig. 9. CDF of MIMO channel capacity for $M=5$ antenna at $6 \mathrm{GHz}$ derived from FEM computations and SME, $N=3$.

DuHamel, R. H. and Isbell, D. E.: Broadband Logarithmically Periodic Antenna Structures, IRE Intern. Conv. Rec., 5, 119-128, 1957.

Foschini, G. J.: Layered space-time architecture for wireless communication in fading environment when using multiple antennas, Bell Labs Tech. J., 41-59, 1996.

Jakes, W. C.: Microwave Mobile Communications, IEEE Press, Inc., New York, 1974.

Kermoal, J. P., Schumacher, L., Pedersen, K. I., Morgensen, P. E., and Frederiksen, F.: A Stochastic MIMO Radio Channel Model With Experimental Validation, 20, 1211-1226, 2002.

Klemp, O. and Eul, H.: Radiation Pattern Analysis of Antenna Systems for MIMO and Diversity Configurations, Adv. Radio Sci., 3, 157-165, 2005.

Klemp, O., Schultz, M., and Eul, H.: A Planar Broadband Antenna for Applications in WLAN and 3G, Proc. of the 2004 Intern. Sympos. Signals, Systems and Electronics ISSSE, Austria, 2004.

Klemp, O., Schultz, M., and Eul, H.: Novel Logarithmically Periodic Antenna Structures for Broadband Polarization Diversity Reception, AEUE, Intern. J. on Electronics and Commun., 59, 268-277, 2005.

Leifer, M. C.: Signal Correlations in Coupled Cell and MIMO antennas, Antennas and Propagation Soc. Intern. Symp., 16-22 June 2002, 3, 194-197, 2002.

Lo, Y. T. and Lee, S. W.: Antenna Handbook - Theory, Applications and Design, Van Nostrand Reinhold Company, New York, 1988.

Narasimhan, M. S., Christopher, S., and Varadarangan, K.: Modal Behavior of Spherical Waves from a Source of EM Radiation with Application to Spherical Scanning, 33, 350-354, 1985.

Rumsey, V. H.: Frequency Independent Antennas, IRE Intern. Conv. Rec., 5, 114-118, 1957.

Stratton, J. A.: Electromagnetic Theory, McGraw Hill, New York, 1941.

Taga, T.: Analysis for Mean Effective Gain of Mobile Antennas in Land Mobile Radio Environments, 39, 117-131, 1990.

Werner, D. H. and Mittra, R.: Frontiers in Electromagnetics, IEEE Press Series on Microwave Technology and RF, New York, 2000.

$\mathrm{Yu}, \mathrm{K}$. and Ottersten, B.: Models for MIMO Propagation Channels, Wiley Journal on Wireless Communications and Mobile Computing, Spec. Issue on Adapt. Antennas and MIMO Syst., 2002. 